\title{
Supporting Information: Large and reconfigurable infrared photothermoelectric effect at oxide interfaces
}

Weitao Dai, ${ }^{\dagger}, \#$ Yi Liang, $,{ }^{\dagger}, \star \#$ Ming Yang, ${ }^{\dagger}$, Dustin Schrecongost, ${ }^{\dagger}$ Prakash Gajurel, ${ }^{\dagger}$ Hyungwoo Lee, ${ }^{\S}$ Jung-Woo Lee, ${ }^{\S}$ Chang-Beom Eom, ${ }^{\S}$ Cheng Cen ${ }^{*}, \uparrow$

${ }^{\dagger}$ Department of Physics and Astronomy, West Virginia University, Morgantown, West Virginia 26506, USA

Guangxi Key Lab for Relativistic Astrophysics, Guangxi Colleges and Universities Key Lab of Novel Energy Materials and Related Technology, School of Physical Science and Technology, Guangxi University, Nanning, Guangxi 530004, China

$\$$ Department of Material Science and Engineering, University of Wisconsin-Madison, Madison, Wisconsin 53706, USA

*Corresponding author: cheng.cen@mail.wvu.edu

\# These authors contributed equally to this work.

\section{I: Experiments}

The photovoltage was measured on a $\mathrm{LAO} / \mathrm{STO}$ sample. 8 unit cells (uc) $\mathrm{LaAlO}_{3}$ thin films were deposited on $\mathrm{TiO}_{2}$ terminated (001) $\mathrm{SrTiO}_{3}$ substrates via pulsed laser deposition (PLD) at $550^{\circ} \mathrm{C}$ under $10^{-3} \mathrm{mbar} \mathrm{O}_{2}$ pressure. After deposition, the sample was cooled to room temperature under an oxygen pressure of 1 mbar. Ohmic contacts to the $\mathrm{LaAlO}_{3} / \mathrm{SrTiO}_{3}$ interface (enclosed by orange boxes in Figure 1(a)) were obtained as following: the electrodes were first patterned via optical lithography; $7 \mathrm{~nm}$ depth of material was milled away in the electrode areas using $\mathrm{Ar}+$ ion etching; etched areas were backfilled with $2 \mathrm{~nm}$ Ti and $6 \mathrm{~nm}$ Au by sputtering. For The metasurface absorber, the similar continuous film was fabricated by photolithography and maintains the electric connection to the $\mathrm{LaAlO}_{3} / \mathrm{SrTiO}_{3}$ interface. Then the cuboid array was fabricated at this film by 
electron beam lithography (EBL) Then the metallic pads for wire bonding were fabricated by ebeam evaporation of $2 \mathrm{~nm} \mathrm{Ti}$ and $30 \mathrm{~nm} \mathrm{Au}$ on top of LAO surface. The e-beam evaporation structures (enclosed by the white dashed lines in Figure 1(a)) overlap partially with the ohmic contacts and the overlapped area is clearly visible in Figure 1(a).

After the fabrication of the metallic structures, the as-grown conductive interface of the $8 \mathrm{uc}$ LAO/STO sample was insulted by oxygen plasma treatment. Then a Hall bar of conductive twodimensional electron gas (2DEG) was created by conductive AFM lithography. Due to the damage of lead 2, an additional conductive channel was written between lead 2 and 5 . The conductive 2DEG pattern was clearly imaged by Kelvin probe force microscope, as shown in the inset of Figure 1(a).

The sample was loaded to a cryostation from Montana Instruments and the scanning photovoltage microscope was performed with a continuous wave incident laser at a wavelength of $830 \mathrm{~nm}$ through a home-made microscope system. The reflection signal (Figure 1(b)) was also monitored during the scan. The diameter of the focused laser point is estimated at $3 \mu \mathrm{m}$ based on the sharpness of the reflection images, which is close to the diffraction limit of the objective lens we used $(20 \times, 0.25 \mathrm{NA})$.

\section{II: Optical absorption of nanocubroid metasurface}

\section{Introduction}

Metasurfaces are ultrathin optical components which have many applications in optics and optoelectronics. Usually, they compose of artificially designed arrays of optical scatters. Here, we

designed a good light metasurface absorber to optimize photovoltage generation and used finite element method (FEM) to simulate its photoabsorption. 


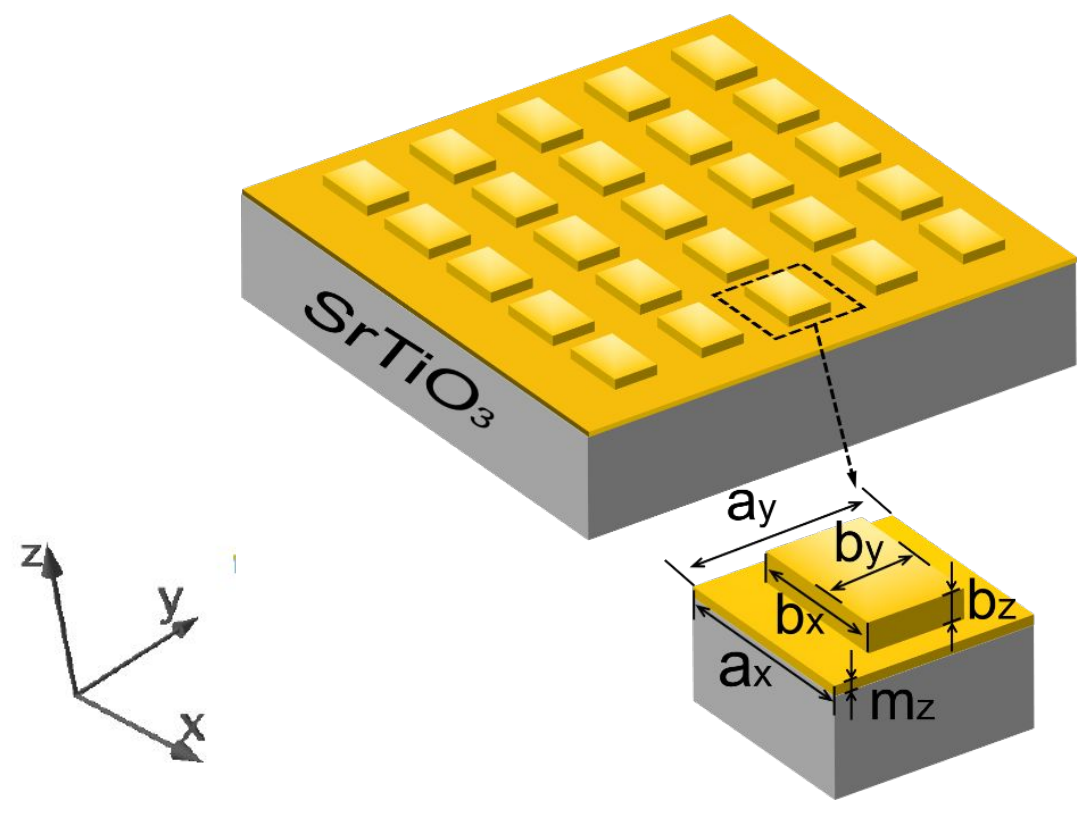

FIG. 1: Scheme of the device design. The blue area represents the $\mathrm{SrTiO}_{3}$ substrate; the golden area represents the gold film with the gold cuboid array.

Figure 1 demonstrates the device design. The grey area represents the $\mathrm{SrTiO}_{3}$ substrate. Since the $\mathrm{LaAlO}_{3}$ layer was very thin and was removed in the area, we neglected it in the electromagnetic wave simulations. The golden area shows the gold nanostructures. Here we consider a simple design: a period nanocuboid array on a continue gold film. The continuous film can be fabricated by photolithography and maintains the electric connection to the $\mathrm{LaAlO}_{3} / \mathrm{SrTiO}_{3}$ interface. Then the cuboid array can be fabricated by one-step e-beam lithography. It is not easy to get high light absorption in the metal nanostructue from top-down approach due to the huge impedance mismatch between metal and air. The continuous film can only absorb $2.5 \%$ light at $800 \mathrm{~nm}$. Metasurface absorber was invented to achieve perfect absorption by using two-layer metal metamaterials. But the absorption happens mainly in the dielectric layer between the two metal layers. 
The coordinate system is also defined in Figure 1. The normal incident light comes from $\mathrm{z}$ direction and the electric field is along $\mathrm{x}$ direction. The nanostructure has six free parameters: the period along $x$ and $y$ direction $a_{\mathrm{x}}$ and $a_{\mathrm{y}}$; the size of the top cuboids $b_{x}, b_{y}$ and $b_{\mathrm{z}}$; the thickness of the gold thin film $m_{\mathrm{z}}$. We try to improve the absorption in the metal structure at $830 \mathrm{~nm}$ by tuning these six geometric parameters as below.

\section{Device optimization}

\section{A. Continuous film}
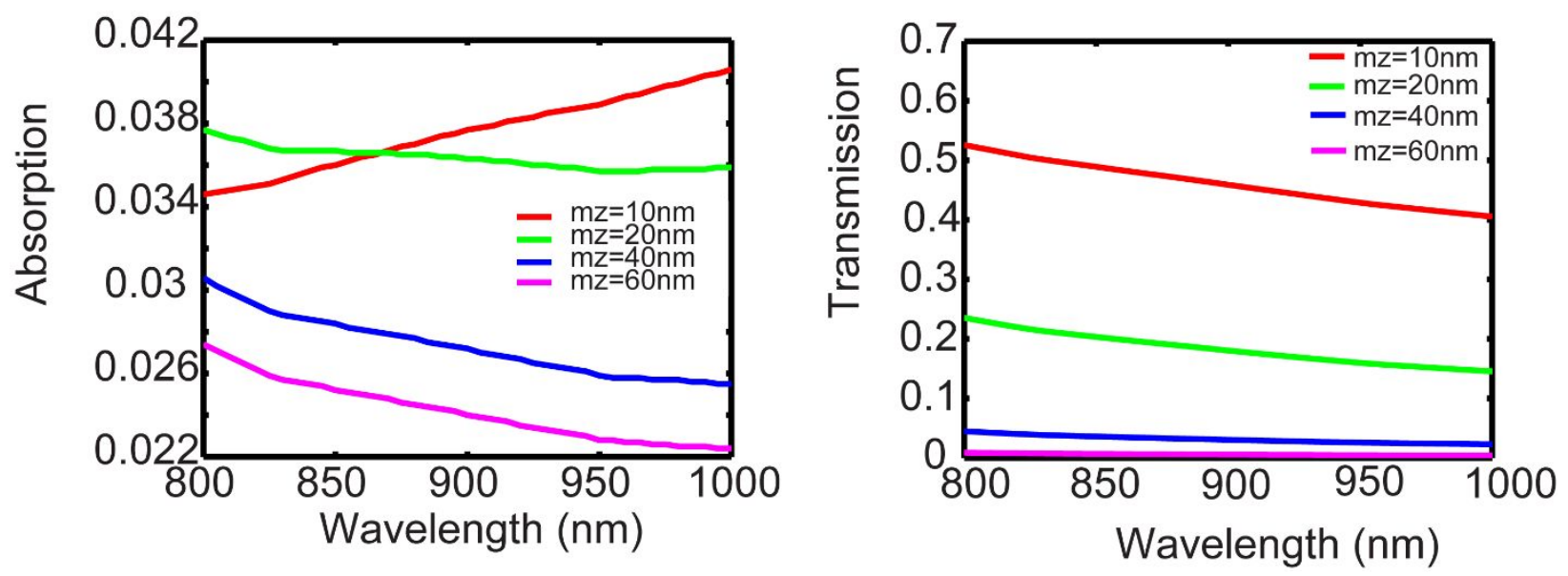

FIG. 2: The absorption and transmission of a continuous gold film on a $\mathrm{SrTiO}_{3}$ substrate.

First, Figure 2 shows the absorption and transmission of a continuous gold film on a $\mathrm{SrTiO}_{3}$ substrate. The absorption is around or less than $4 \%$ for various thicknesses. The transmission is not negligible when the film is thinner than $40 \mathrm{~nm}$. We have to consider the reflection from the gold $/ \mathrm{SrTiO}_{3}$ interface and the interference effect. This explains the thinner film has higher absorption. 


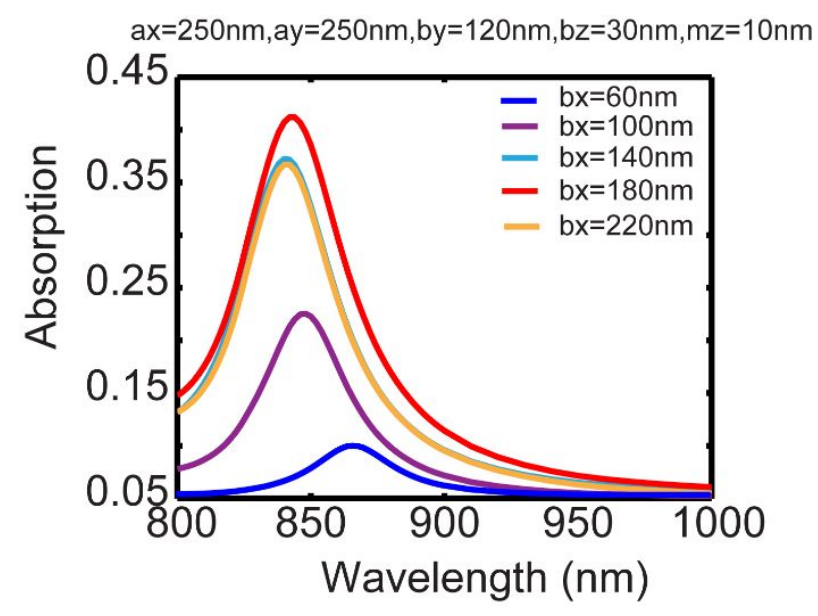

Fig. 3: The absorption curves at various $b_{x}$

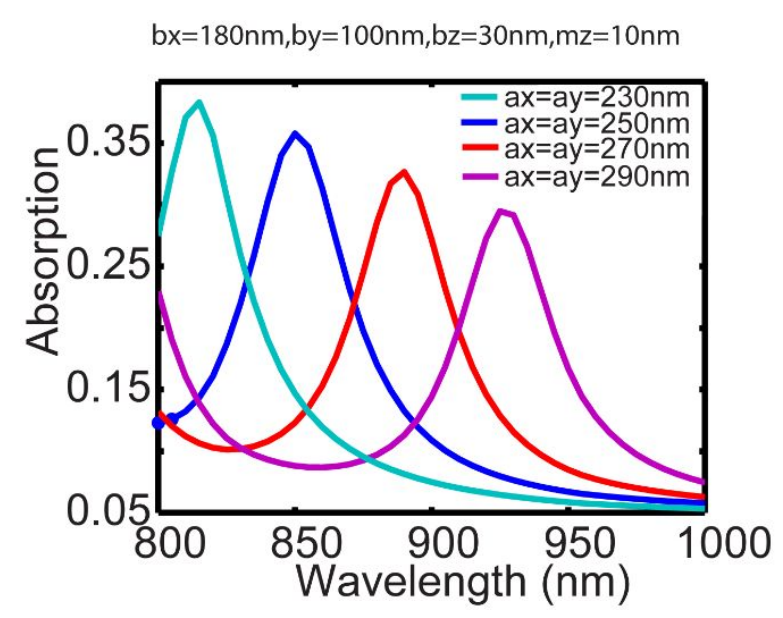

Fig.4: The absorption curves at various $a_{x}$

\section{B. Study of $b_{x}$}

The simulation results of the absorption curves at various $b_{x}$ are shown in Figure 3 . The period of the structures is $a_{x}=a_{y}=250 \mathrm{~nm}$, and $b_{\mathrm{x}}$ changes from $60 \mathrm{~nm}$ to a value close to the period. The period is chosen by considering the EBL capability and the size of the focused incident light. The focused light spot has diameter around $3 \mu \mathrm{m}$ and covers more than 10 periods of the nanostructure. So we can model the incident wave as a plane wave. The thickness of the bottom continuous film is $m_{\mathrm{z}}=10 \mathrm{~nm}$. In the actual devices, the continuous film contains a Ti film and an Au film, with total thickness around $10 \mathrm{~nm}$. We will study the continuous film thickness later. The metal cuboid has thickness $b_{\mathrm{z}}=30 \mathrm{~nm}$.

From Figure 3, we found $b_{x}$ not only change the absorption but also change the resonant wavelength for the largest absorption. However, when $b_{x}>140 \mathrm{~nm}$, the wavelengths of the absorption peaks don't change much with $b_{\mathrm{x}}$. Furthermore, one can see that $b_{x}=180 \mathrm{~nm}$ show the largest absorption peak, indicating that there is a best $b_{x}$ for our metasurface absorber to realize largest absorption peak. It could not be too small or too large. 


\section{Study of $a_{x}$}

Figure 4 demonstrates the absorption curves at various $a_{x}$. We can see clearly the peaks move with the period. The peaks move from $815 \mathrm{~nm}$ to $925 \mathrm{~nm}$ when the period changes from $230 \mathrm{~nm}$ to 290 $\mathrm{nm}$. So, the parameter $a_{x}$ decides the resonant wavelength for the largest absorption. When $a_{x}$ increases, resonant wavelength move to larger value. Moreover, $a_{x}$ is more important than $b_{x}$ to tune the absorption peak to desired wavelength.

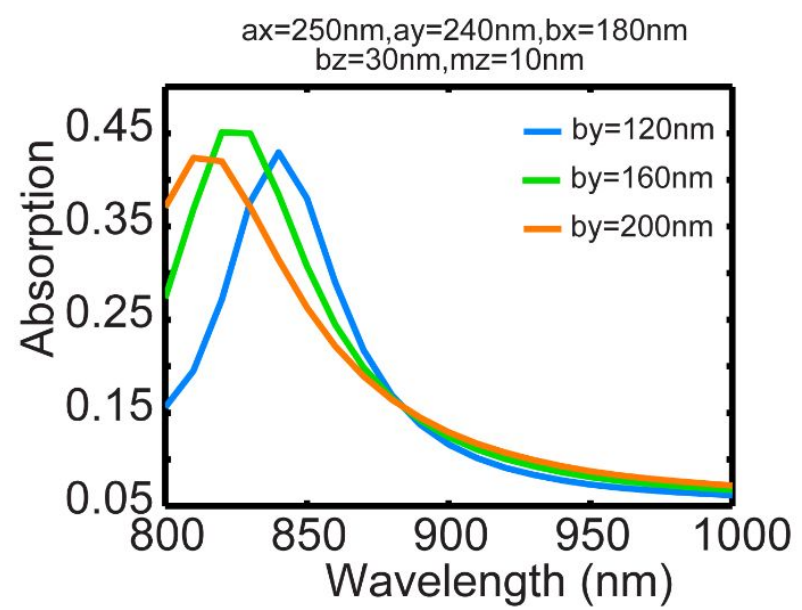

Fig. 5: The absorption curves at various $b_{y}$

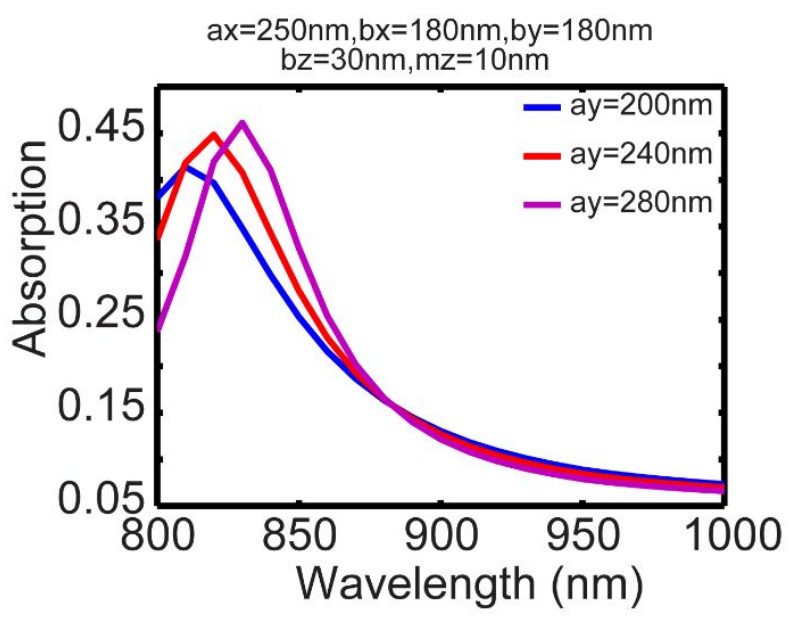

Fig. 6: The absorption curves at various $a_{y}$

\section{Study of $a_{y}$ and $b_{y}$}

Now we begin to study the geometry parameters along y direction: $a_{y}$ and $b_{y}$. The rest parameters are: $a_{x}=250 \mathrm{~nm}, b_{x}=180 \mathrm{~nm}, b_{z}=30 \mathrm{~nm}$ and $m_{\mathrm{z}}=10 \mathrm{~nm}$. In Figure 5, $a_{y}=240 \mathrm{~nm}$ is fixed and $b_{y}$ changes from $120 \mathrm{~nm}$ to $200 \mathrm{~nm}$; in Figure $6, b_{y}=180 \mathrm{~nm}$ is fixed and $a_{y}$ changes from $200 \mathrm{~nm}$ to $280 \mathrm{~nm}$. Both figures demonstrate the absorption spectra are also sensitive to the geometry parameters along y direction. When $a_{y}$ is fixed, the peak wavelength moves to shorter wavelength when $b_{y}$ increases, the same as Fig.3. When $b_{y}$ is fixed, the peak wavelength moves to longer wavelength when $a_{y}$ increases. 


\section{E. Optimization at $\lambda=830 \mathrm{~nm}$}

Figure 7 and 8 demonstrate the absorption of various nanostructures at $\lambda=830 \mathrm{~nm}$. Figure 7 conveys similar messages as previous discussion: the absorption is sensitive to ax and not very sensitive to $b_{x}$. Next, we choose $a_{x}=250 \mathrm{~nm}, b_{x}=170 \mathrm{~nm}$, and study how $a_{y}$ and $b_{y}$ change the absorption in Figure 8. We found the absorption is sensitive to the difference between $a_{y}$ and $b_{y}$; Clearly, when the difference is near $90 \mathrm{~nm}$, metasurface absorber shows the largest absorption. In this case we have multiply choices to achieve good absorption.

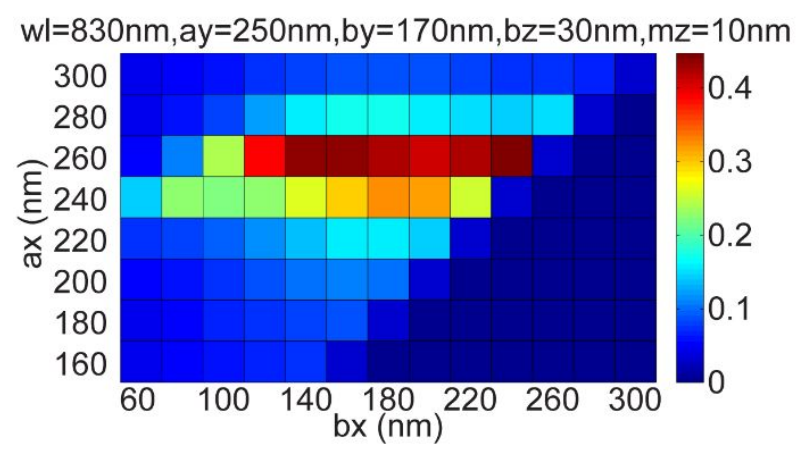

FIG. 7: Absorption at $\lambda=830 \mathrm{~nm} . a_{y}=250$ $\mathrm{nm}$ and $b_{y}=170 \mathrm{~nm}$ are fixed with various $a_{x}$ and $b_{x}$ combinations.

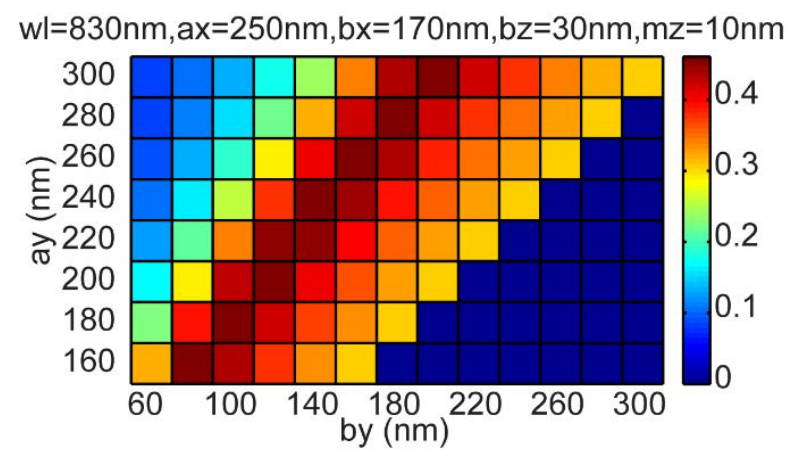

FIG. 8: Absorption at $\lambda=830$ nm. $a_{x}=250$ $\mathrm{nm}$ and $b_{x}=170 \mathrm{~nm}$ are fixed with various $a_{x}$ and $b_{x}$ combinations

\section{F. Optimization of the thickness}

Let's study the thickness of continuous film under the nanocuboid array first. Figure 8 demonstrates $m_{\mathrm{z}}$ is very important parameters. $2 \mathrm{~nm}$ increase of the thickness results significant change of the absorption spectra including the resonant wavelength and the absorption peak value. The absorption became weaker with the increasing thickness $m_{\mathrm{z}}$. Actually, here, we have to consider 
the coupling of the $\mathrm{Au} / \mathrm{SrTiO}_{3}$ interface and $\mathrm{Au} /$ air interface. The plasmonic modes supported by the thin metal film are very sensitive to the film thickness due to the coupling. So, the coupling from the $\mathrm{Au} / \mathrm{SrTiO}_{3}$ interface is crucial to get high absorption.

Next, we study the influence of $b_{\mathrm{z}}$, the thickness of the cuboids. Figure 10 shows how the absorption spectra change with $b_{\mathrm{z}}$ at $m_{\mathrm{z}}=10 \mathrm{~nm}$. Generally, the larger the thickness, the larger the absorption. But the curves in the same figure have similar shapes and $b_{z}$ cannot move the resonant wavelength a lot. The fact that the resonant wavelength is not very sensitive to the cuboid shape $\left(b_{x}, b_{y}\right.$ and $\left.b_{z}\right)$ suggests that the resonance comes from the plasmonic modes supported by the air/gold $/ \mathrm{SrTiO}_{3}$ multilayer structure and the cuboid array is used to excite the plasmonic modes.

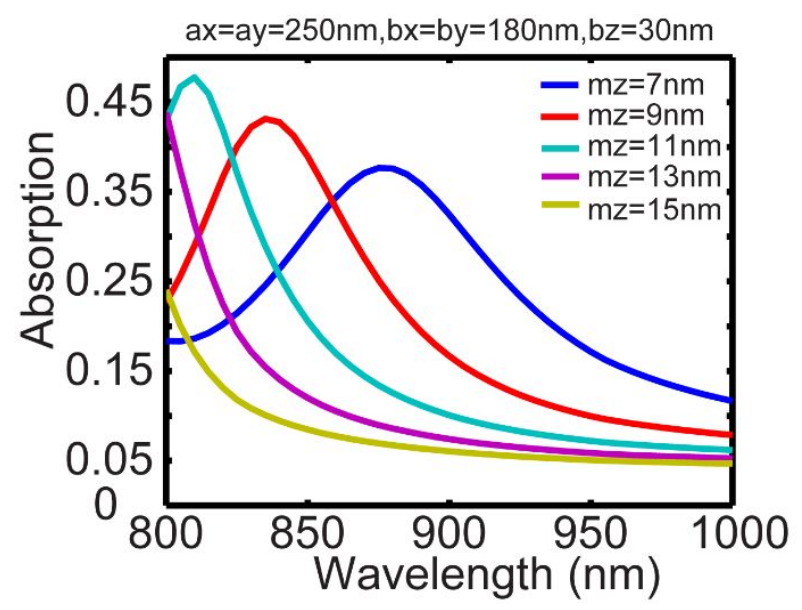

FIG. 9: Study of $m_{z}$, the thickness of

continuous film under the nanocuboid array.

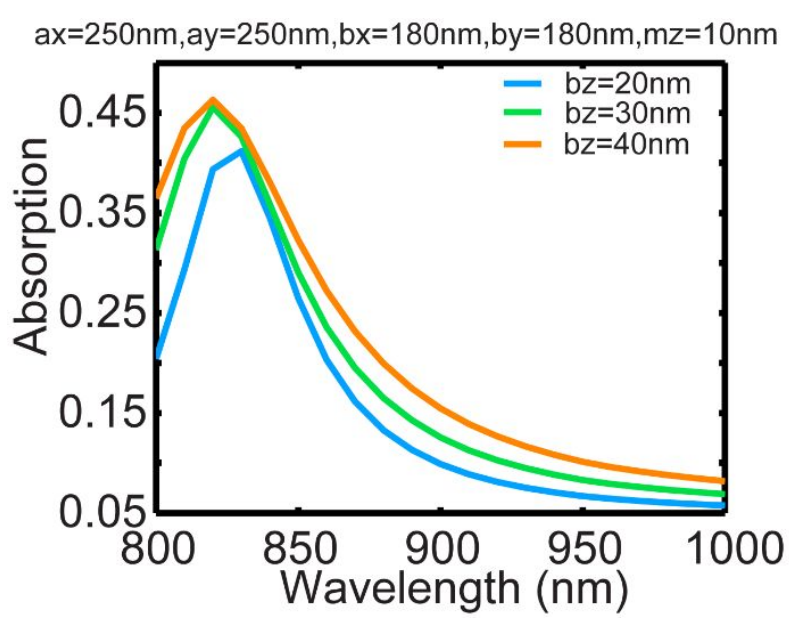

FIG. 10: Study of $b_{z}$, the thickness of the cuboids.

In conclusion, we have studied the influence of the six parameters of our metasurface absorber on photoabsorption via simulation. From these results, for our experiment, we can choose appropriate parameters to realize a strong absorption to improve photovoltage generation. 